Proyecciones

Vol. 23, No 3, pp. 235-240, December 2004.

Universidad Católica del Norte

Antofagasta - Chile

\title{
UNIFORM BOUNDEDNESS IN VECTOR - VALUED SEQUENCE SPACES
}

\author{
CHARLES SWARTZ \\ New Mexico State University, USA \\ Received: July 2004. Accepted : November 2004
}

\begin{abstract}
Let $\mu$ be a normal scalar sequence space which is a K-space under the family of semi-norms $M$ and let $X$ be a locally convex space whose topology is generated by the family of semi-norms $X$. The space $\mu\{X\}$ is the space of all $X$ valued sequences $x=\left\{x_{k}\right\}$ such that $\left\{q\left(x_{k}\right)\right\} \in$ $\mu\{X\}$ for all $q \in X$. The space $\mu\{X\}$ is given the locally convex topology generated by the semi-norms $\pi_{p q}(x)=p\left(\left\{q\left(x_{k}\right)\right\}\right), p \in X, q \in M$. We show that if $\mu$ satisfies a certain multiplier type of gliding hump property, then pointwise bounded subsets of the $\beta$-dual of $\mu\{X\}$ with respect to a locally convex space are uniformly bounded on bounded subsets of $\mu\{X\}$.
\end{abstract}




\section{Introduction}

In [Sw3], in order to establish uniform boundedness results for sequence spaces, we introduced a gliding hump property in which the "humps" in the sequence space are multiplied by elements of another sequence space in order to facilitate the convergence of a series whose elements are those composed of the "humps". In this note we show that this gliding hump property can be employed to establish uniform boundedness results for the operator valued $\beta$-dual of certain vector-valued sequence spaces.

We describe the vector-valued sequence spaces which will be considered. Let $\mu$ be a normal scalar-valued sequence space containing $c_{00}$, the space of all sequences which are eventually 0 , and which is a Hausdorff locally convex K-space whose topology is generated by the family of semi-norms $\mathcal{M}$. Let $X(Y)$ be a Hausdorff locally convex space whose topology is generated by the family of semi-norms $\mathcal{X}(\mathcal{Y})$ and let $L(X, Y)$ be the space of all continuous linear operators from $X$ into $Y$. Let $\mu\{X\}$ be the space of all $X$-valued sequences such that $\left\{q\left(x_{k}\right)\right\} \in \mu$ for every $q \in \mathcal{X}$. Since $\mu$ is normal, $\mu\{X\}$ is a vector space and we supply $\mu\{X\}$ with the locally convex topology generated by the family of semi-norms $\pi_{p q}(x)=p\left(\left\{q\left(x_{k}\right)\right\}\right)$ for $x=\left\{x_{k}\right\} \in \mu\{X\}, p \in \mathcal{M}, q \in \mathcal{X}$. For perfect sequence spaces these spaces were introduced by Pietsch $([\mathrm{P}])$ and considered in $[\mathrm{Ro}],[\mathrm{F}],[\mathrm{FP}]$ and $[\mathrm{Sw} 3]$; they include such spaces as the space of absolutely $p$ th power summable series $l^{p}\{X\}$. One of the basic problems in this area is to determine which properties such as barrelledness of $\mu\{X\}$ are inherited from $\mu$ and $X$. For example, sufficient conditions for quasi-barrelledness and barrelledness are given in $[\mathrm{FP}],[\mathrm{F}]$. The $\beta$-dual of $\mu\{X\}$ with respect to $Y, \mu\{X\}^{\beta Y}$, is defined to be all sequences $T=\left\{T_{k}\right\} \subset L(X, Y)$ such that $\sum_{k=1}^{\infty} T_{k} x_{k}$ converges for every $x=\left\{x_{k}\right\} \in \mu\{X\}$ ([BL]); if $T \in \mu\{X\}^{\beta Y}$ and $x \in \mu\{X\}$, we write $T \cdot x=\sum_{k=1}^{\infty} T_{k} x_{k}$ and if $A \subset \mu\{X\}, B \subset \mu\{X\}^{\beta Y}$, , we write $B \cdot A=\{T \cdot x: T \in B, x \in A\}$. We consider sufficient conditions for a family $B \subset \mu\{X\}^{\beta Y}$ which is pointwise bounded on $\mu\{X\}$ to be uniformly bounded on bounded subsets of $\mu\{X\}$,i.e., we seek a uniform boundedness or Banach-Mackey principle for the pair $\mu\{X\}, \mu\{X\}^{\beta Y}$.

\section{Main Result}

We first establish a lemma which will be used in the proof of our main result. The pair $(X, Y)$ is said to satisfy the property UB (Uniform Boundedness) if for every family $F \subset L(X, Y)$ which is pointwise bounded on $X$ is 
uniformly bounded on bounded subsets of $X$ ([Sw2]12.5). For example, if $X$ is barrelled, then any pair $(X, Y)$ has UB by the Uniform Boundedness Principle for barrelled spaces ([Sw1]24.11, [ Wi] 9.3.4). An interval in $\mathbf{N}$ is a subset of the form $[m, n]=\{k: m \leq k \leq n\}, m, n \in \mathbf{N}, m \leq n$; a sequence $\left\{I_{k}\right\}$ of intervals is increasing if $\max I_{k}<\min I_{k+1}$. If $I$ is an interval, the characteristic function of $I$ is denoted by $C_{I}$ and if $x=\left\{x_{k}\right\}$ is an $X$-valued sequence, then $C_{I} x$ will denote the coordinatewise product of $C_{I}$ and $x$.

Lemma 1. Assume that $a_{k}>0$ and $a_{k+1} \geq a_{k}$ for every $k$ and that $(X, Y)$ satisfies UB. If $B \subset \mu\{X\}^{\beta Y}$ is pointwise bounded on $\mu\{X\}, A \subset$ $\mu\{X\}$ is coordinatewise bounded and $B \cdot A$ is unbounded in $Y$, then there exist a continuous semi-norm $r$ on $Y,\left\{T^{k}\right\} \subset B,\left\{x^{k}\right\} \subset A$ and increasing intervals $\left\{I_{k}\right\}$ such that $r\left(T^{k} \cdot C_{I_{k}} x^{k}\right)>a_{k}$.

Proof : If the conclusion fails, there is a continuous semi-norm $r$ on $Y$, $x^{k} \in A, T^{k} \in B$ such that $r\left(T^{k} \cdot x^{k}\right)>a_{k}+k$. Put $k_{1}=1$. There exists $n_{1}$ such that $r\left(\sum_{j=1}^{n_{1}} T_{j}^{k_{1}} x_{j}^{k_{1}}\right)>a_{1}+1$. Now for every $j,\left\{x_{j}^{k}: k\right\}$ is bounded by hypothesis and since $c_{00}\{X\} \subset \mu\{X\},\left\{T_{j}^{k}: k\right\}$ is pointwise bounded on $X$. By the UB property, $\left\{T_{j}^{k} x_{j}^{k}: k\right\}$ is bounded in $Y$. There exists $k_{2}>k_{1}$ such that $\sum_{j=1}^{n_{1}} \frac{1}{k_{2}} r\left(T_{j}^{k_{2}} x_{j}^{k_{2}}\right)<1$. Hence, $r\left(\sum_{j=n_{1}+1}^{\infty} T_{j}^{k_{2}} x_{j}^{k_{2}}\right)>a_{k_{2}}$. Pick $n_{2}>n_{1}$ such that $r\left(\sum_{j=n_{1}+1}^{n_{2}} T_{j}^{k_{2}} x_{j}^{k_{2}}\right)>a_{k_{2}}$. Set $I_{2}=\left[n_{1}+1, n_{2}\right]$. Now just continue this construction and relabel.

A scalar version of this lemma is given in [Sw3].

Our main result involves the gliding hump properties introduced in [Sw $3]$. Let $\lambda$ be a scalar-valued sequence space which contains $c_{00}$. For the space $\mu\{X\}$ these are given in the following definitions.

Definition 2. $\mu\{X\}$ has the strong $\lambda$ gliding hump property (strong $\lambda$-GHP) if whenever $\left\{I_{k}\right\}$ is an increasing sequence of intervals and $\left\{x^{k}\right\}$ is a bounded sequence in $\mu\{X\}$, then for every $t=\left\{t_{k}\right\} \in \lambda$ the coordinate sum of the series $\sum t_{k} C_{I_{k}} x^{k}$ belongs to $\mu\{X\}$.

Definition 3. $\mu\{X\}$ has the weak $\lambda$ gliding hump property (weak $\lambda$ GHP) if whenever $\left\{I_{k}\right\}$ is an increasing sequence of intervals and $\left\{x^{k}\right\}$ is a bounded sequence in $\mu\{X\}$, there is a subsequence $\left\{n_{k}\right\}$ such that the coordinate sum $\sum t_{k} C_{I_{n_{k}}} x^{k}$ belongs to $\mu\{X\}$.

Examples of spaces satisfying both strong and weak $\lambda$-GHP are given in [Sw3]. For example, $l^{p}$ has strong $l^{p}$-GHP for $0<p \leq \infty$ and $l^{\infty}$ and $c_{0}$ have strong $c_{0}$-GHP. 
The proof of our main result employs a matrix theorem due to Antosik and Mikusinski which we now state for the convenience of the reader.

Theorem 4. Let $M=\left[y_{i j}\right]$ be an infinite matrix with entries from $Y$. Suppose

(1) $\lim _{i} y_{i j}=0$ for every $j$,

(2) for every increasing sequence of positive integers $\left\{m_{j}\right\}$ there is a subsequence $\left\{n_{j}\right\}$ of $\left\{m_{j}\right\}$ such that $\sum_{j=1}^{\infty} y_{i n_{j}} \rightarrow 0$,

then $y_{i i \rightarrow 0}$.

For a proof (of a more general result), see [Sw 2],2.2.2. A matrix satisfying the conditions of Theorem 4 is called a $K$-matrix and Theorem 4 is called the Antosik-Mikusinski Diagonal Theorem.

Theorem 5. Assume that $\lambda$ contains a vector $\left\{b_{k}\right\}$ with $b_{k}>0$ for every $k$. Assume that $\mu$ has strong $\lambda$-GHP and that $(X, Y)$ has UB. If $A \subset \mu\{X\}$ is bounded and $B \subset \mu\{X\}^{\beta Y}$ is pointwise bounded on $\mu\{X\}$, then $B \cdot A$ is bounded.

Proof : Suppose the conclusion fails. Since $\mu$ is a K-space, $A$ is coordinatewise bounded so we may apply Lemma 1 with $a_{k}=k / b_{k}$. Let the notation be as in Lemma 1 and define the matrix $M$ by $M=\left[m_{i j}\right]=$ $\left[T_{i} / i \cdot b_{j} C_{I_{j}} x^{j}\right]$. We claim that $M$ is a $K$-matrix in the sense of Antosik and Mikusinski . First, the columns of $M$ converge to 0 by the pointwise boundedness of $B$. Let $z=\left\{z_{i}\right\}$ be the coordinatewise sum of the series $z=\sum_{j=1}^{\infty} b_{j} C_{I_{j}} x^{j}$. We claim that $z \in \mu\{X\}$. Let $q \in \mathcal{X}$. By the strong $\lambda$-GHP the series $\sum_{j=1}^{\infty} \sum_{k \in I_{j}} b_{j} q\left(x_{k}^{j}\right) e^{k}$, where $e^{k}$ is the sequence with a 1 in the $k$ th coordinate and 0 in the other coordinates, converges in $\mu$ to the element $\left\{q\left(z_{i}\right)\right\} \in \mu$. That is, $z \in \mu\{X\}$. Therefore, $\sum_{j=1}^{\infty} m_{i j}=\sum_{j=1}^{\infty} T_{i} / i \cdot b_{j} C_{I_{j}} x^{j}=T_{i} / i \cdot z \rightarrow 0$ by the pointwise boundedness of $B$. Since the same argument can be applied to any subsequence, $M$ is a $K$-matrix. By the Antosik-Mikusinski Diagonal Theorem the diagonal of $M$ converges to 0 . But this contradicts the conclusion of Lemma 1 since $r\left(m_{i i}\right)>1$.

Remark 6. The proof above also applies if $\mu$ has the weak $\lambda$-GHP and $X$ is normed since in this case it is only necessary to check a single element $q \in \mathcal{X}$ to establish that $z \in \mu\{X\}$.

Remark 7. The assumption that the pair has the UB property is necessary in Theorem 5. For assume that $F \subset L(X, Y)$ is a subset which is pointwise bounded on $X$ and let $A \subset X$ be bounded. If $x \in X, T \in L(X, Y)$, 
put $x^{1}=(x, 0,0, \ldots), T^{1}=(T, 0,0, \ldots)$ and $A^{1}=\left\{x^{1}: x \in A\right\}, F^{1}=\left\{T^{1}\right.$ : $T \in F\}$. Then $A^{1} \subset \mu\{X\}, F^{1} \subset \mu\{X\}^{\beta Y}$ and $F^{1} \cdot A^{1}=\{T x: T \in F, x \in$ $A\}=F A$ so $F^{1}$ is pointwise bounded on $\mu\{X\}$. Thus, if the conclusion of Theorem 5 holds, then $F A$ is bounded so the pair $(X, Y)$ would have UB.

Remark 8. Without some assumption such as the GHP, the conclusion of Theorem 5 cannot hold. For, let $X$ be a non-barrelled normed space and let $\left\{x_{k}: k\right\} \subset X$ be bounded in $X$ and let $B=\left\{y_{k}: k\right\} \subset X^{\prime}$ be weak* bounded in $X^{\prime}$ with $\sup \left\{\left|\left\langle y_{k}, x_{k}\right\rangle\right|: k\right\}=\infty$. Set $A^{1}=\left\{x_{k} e^{k}: k\right\}$ and $B^{1}=\left\{y_{k}^{1}=\left(y_{k}, y_{k}, \ldots\right): k\right\}$. Then $A^{1} \subset c_{00}\{X\}$ and $B^{1} \subset c_{00}\{X\}^{\beta \mathbf{R}}$, $B^{1}$ is pointwise bounded , $c_{00}\{X\}$ does not have $l^{1}$-GHP and $B^{1} \cdot A^{1}=$ $\left\{\left\langle y_{k}, x_{k}\right\rangle: k\right\}$ is unbounded so the conclusion of Theorem 5 fails to hold.

A scalar version of Theorem 5 is given in [Sw3 ], Corollary 7; however the proof of this result in [Sw3] relies on duality methods and cannot be employed to obtain the operator-valued version given in Theorem 5 . The Antosik-Mikusinski diagonal Theorem is used in place of the duality methods. The assumptions on $\mu$ are also somewhat stronger in [Sw3]. Another scalar Banach-Mackey type result is given in $[\mathrm{F}] 3.7$.

\section{References}

[1] J. Boos and T. Leiger, Some distinguished subspaces of domains of operator valued matrices, Results Math., 16, pp. 199-211, (1989).

[2] M. Florencio and P. Paul, Barrelledness conditions on certain vector valued sequence spaces, Arch. Math., 48, pp. 153-164, (1987).

[3] J. Fourie, Barrelledness Conditions on Generalized Sequence Spaces, South African J. Sci., 84, pp. 346-348, (1988).

[4] A. Pietsch, Verallgemeinerte vollkommene Folgenraume, Schrift. Inst. Math., Deutsch Akad. Wiss. Berlin, Heft 12, Acad. Verlag, Berlin, (1962).

[5] R. Rosier, Dual Spaces of Certain Vector Sequence Spaces, Pacific J. Math., 46, pp. 487-501, (1973). 
[6] C. Swartz, Introduction to Functional Analysis, Marcel Dekkar, N. Y., (1992).

[7] C. Swartz, Infinite Matrices and the Gliding Hump, World. Sci. Publ., Singapore, (1996).

[8] C. Swartz, A Multiplier Gliding hump Property for Sequence Spaces, Proyecciones Revista de Matemática, Vol. 20, pp. 19-31, (2001).

[9] A. Wilansky, Modern Methods in Topological Vector Spaces, McGrawHill, N. Y., (1978).

\section{Charles Swartz}

Department of Mathematical Sciences

New Mexico State University

Las Cruces, NM 88003

USA

e-mail : cswartz@nmsu.edu 